\title{
Determination of Oxygen Diffusion Coefficient of Poly(methacrylonitrile) II and the Calculation of Diffusion Activation Energy
}

\author{
T. Y. KATIRCIOGLU, ${ }^{1 *}$ H. Y. KAPTAN, ${ }^{2}$ O. GUVEN $^{3}$ \\ ${ }^{1}$ Department of R\&D, MND (MSB. ARGE D.), Yucetepe, Ankara, 06650 Turkey \\ ${ }^{2}$ Department of Physics Engineering, Beytepe, Ankara, 06532 Turkey \\ ${ }^{3}$ Department of Chemistry, Beytepe, Ankara, 06532 Turkey
}

Received 15 July 1998; accepted 26 January 1999

\begin{abstract}
Poly(methacrylonitrile) (PMAN) samples in the shape of a cylinder used in this study were obtained from methacrylonitrile by bulk polymerization. The electron spin resonance (ESR) method has been used to calculate oxygen diffusion coefficient $(D)$ into PMAN samples exposed to high-energy radiation at different doses in vacuum by observing the ESR radical signal change. In order to calculate the dose-independent diffusion coefficient $\left(D_{0}\right), \ln D$ values were plotted against dose values. The low dose region of this curve was extrapolated to a zero-dose value, and $D_{0}$ was calculated as $D_{0}$ $=3.1 \times 10^{-9} \mathrm{~cm}^{2} / \mathrm{s}$. Although $D_{0}$ values were in very good agreement with the one obtained for the thin-film sample of PMAN, the dose dependence of the oxygen diffusion into the cylindrical PMAN samples was observed as being converse of the thin-film of PMAN, ${ }^{1}$ as expected, because of the big difference of the surface/mass values between relatively big cylindrical PMAN samples and thin-film of PMAN samples. The activation energy $\left(E_{a}\right)$ values of the oxygen diffusion into PMAN were calculated as $E_{a 1}$ $=27.9 \mathrm{~kJ} / \mathrm{mol}$ for the $20-60^{\circ} \mathrm{C}$ temperature range $E_{\alpha 2}=74.2 \mathrm{~kJ} / \mathrm{mol}$ for the temperatures above $60^{\circ} \mathrm{C}$ of the $36 \mathrm{kGy}$ gamma-irradiated samples. The temperature value of the break point of activation energy was near to the $T_{g}$ of PMAN. ${ }^{2}$ ○ $1999 \mathrm{John}$ Wiley \& Sons, Inc. J Appl Polym Sci 74: 1108-1118, 1999
\end{abstract}

Key words: poly(methacrylonitrile); oxygen; diffusion; activation energy

\section{INTRODUCTION}

Diffusion into polymers depends mostly on the thermal movement of the polymer chain and end groups as well as the free volume in the polymer to be used by diffusive material and all other changes in the structure, which can affect the above-mentioned properties, like side groups, polarity, plasticizers, and interchain interac-

Correspondence to: T. Y. Katircioglu.

Journal of Applied Polymer Science, Vol. 74, 1108-1118 (1999)

(ㄷ) 1999 John Wiley \& Sons, Inc.

CCC 0021-8995/99/051108-11 tions. ${ }^{3-6}$ In the case of the diffusion into the irradiated polymer, the shape of the sample from the point of the surface /mass ratio value affects the diffusion velocity and dose diffusion relation. ${ }^{1,7}$

When the polymers are irradiated with highenergy radiation, such as gamma rays, free radicals are produced in the polymer. The termination or decay of these radicals depend mainly on the temperature and availability of the oxygen molecules or other diffusive materials that may scavenge these radicals. If the room temperature is much lower than the glassy transition temperature $\left(T_{g}\right)$ of the polymer, generally, molecular 
thermal movement at room temperature is not enough for the termination reaction between the macro radicals formed from high-energy radiation. As long as these samples keep under vacuum, there will be no change on their electron spin resonance (ESR) spectrums. When these vacuum-irradiated samples are exposed to air, the radicals in the polymer are trapped by oxygen and transformed into peroxy radicals by the addition reaction shown as follows:

$$
\sim \mathrm{R}^{\bullet}+\mathrm{O}_{2} \rightarrow \sim \mathrm{ROO}^{\bullet}
$$

The ESR signals' values that come from the radicals in the structure start to decrease their intensities, and the shape of the spectrum totally changes to a peroxy signal at last. This effect can be directly used to measure the oxygen uptake of irradiated polymer samples. ${ }^{1,7,8}$ But if there is a radical self-decay reaction, especially at a higher temperature study, in order to use the ESR techniques for diffusion investigation, the following termination reactions regarding self-decay of the radicals should be considered:

$$
\sim \mathrm{R}^{\bullet}+\sim \mathrm{R}^{\bullet} \underset{\text { combination }}{\longrightarrow} \sim \text { One longer polymer chain }
$$

$\sim \mathrm{R}^{\bullet}+\sim \mathrm{R}^{\bullet} \underset{\mathrm{H} \text { transfer }}{\longrightarrow} \sim+\sim$ Two polymer chains

and the consumption rate of radicals resulted from only oxygen or other diffusive material can be calculated as the difference of the termination reactions between in air and in vacuum at each temperature.

\section{EXPERIMENTAL}

Poly(methacrylonitrile) (PMAN) samples were obtained from methacrylonitrile (Merck) by bulk polymerization after getting passed through active alumina to remove the inhibitors from the monomer. The concentration of $\alpha, \alpha$-azobisizobutyronitrile (AIBN) as the initiator was $20 \mathrm{mg} / 10$ $\mathrm{mL}$ MAN. The polymerization was done in a sealed Pyrex tube under nitrogen atmosphere with a radius $r$ equal to $0.185 \mathrm{~cm}$. The polymerization reaction was continued in the $30^{\circ} \mathrm{C}$ heated-water bath for $100 \%$ conversion for 75 days.
The conversion could be followed by the phase difference of the polymer that was not soluble in its monomer. Before usage of the polymer samples, they were kept under vacuum for 7 days at $35^{\circ} \mathrm{C}$ to remove residue of the monomer and unreacted radicals.

After having been kept under vacuum at $35^{\circ} \mathrm{C}$, the samples, which have a height $h$ equal to $6 \mathrm{~cm}$ and a radius $r$ equal to $0.185 \mathrm{~cm}$ were formed by cutting from the long cylindrical sample.

Before gamma irradiation, samples were placed into pyrex tubes, and they were sealed by flame after keeping for $1 \mathrm{~h}$ under vacuum less than $10^{-6} \mathrm{~mm} \mathrm{Hg}$, obtained with the serial connection of mechanical dry and oil pomps.

\section{INSTRUMENTATION}

The samples were irradiated in a Gamma Cell 220-type ${ }^{60}$ Co $\gamma$ irradiater at room temperature, under vacuum and at a dose rate of $0.87 \mathrm{kGy} / \mathrm{h}$. Dose rates calculated with the standard Fricke ${ }^{9}$ dosimetry method. The paramagnetic centers produced by high-energy radiation were removed by carefully heating of the inverted ends of the tubes, and they were placed in the cavity of the ESR spectrometer. During the heating operation, the samples were kept in liquid nitrogen to prevent them from the heating that may cause radical self-decay in the sample.

ESR spectrums of the samples irradiated were taken with a Varian E-9 type X Band spectrometer under vacuum and after conduct with air. The spectra were taken and recorded at room temperature. The microwave power was kept at $1.5 \mathrm{mV}$ throughout the study. As radical concentration $(R)$, the height of the selected peak with an asterisk in Figure 1 was directly used because there was no important change on the width of this peak, and it was not overlapped with the peroxy radical ESR peak. The intensity of the spectrum was decreased very slowly at room temperature, so the duration to take ESR spectrums were extended from $30 \mathrm{~min}$ to the day time to see the difference in the ESR spectrum. For higher temperature, the time intervals between two ESR spectrum was shortened. Just after taking each ESR spectrum of PMAN sample, the ESR spectrum of $\propto$, $\propto$,diphenyl $\beta$-picrylhydrazyl (DPPH) sample as a standard was taken to normalize the signal values of PMAN samples.

Differential scanning calorimetry (DSC) measurement were carried out by using Dupont DSC- 


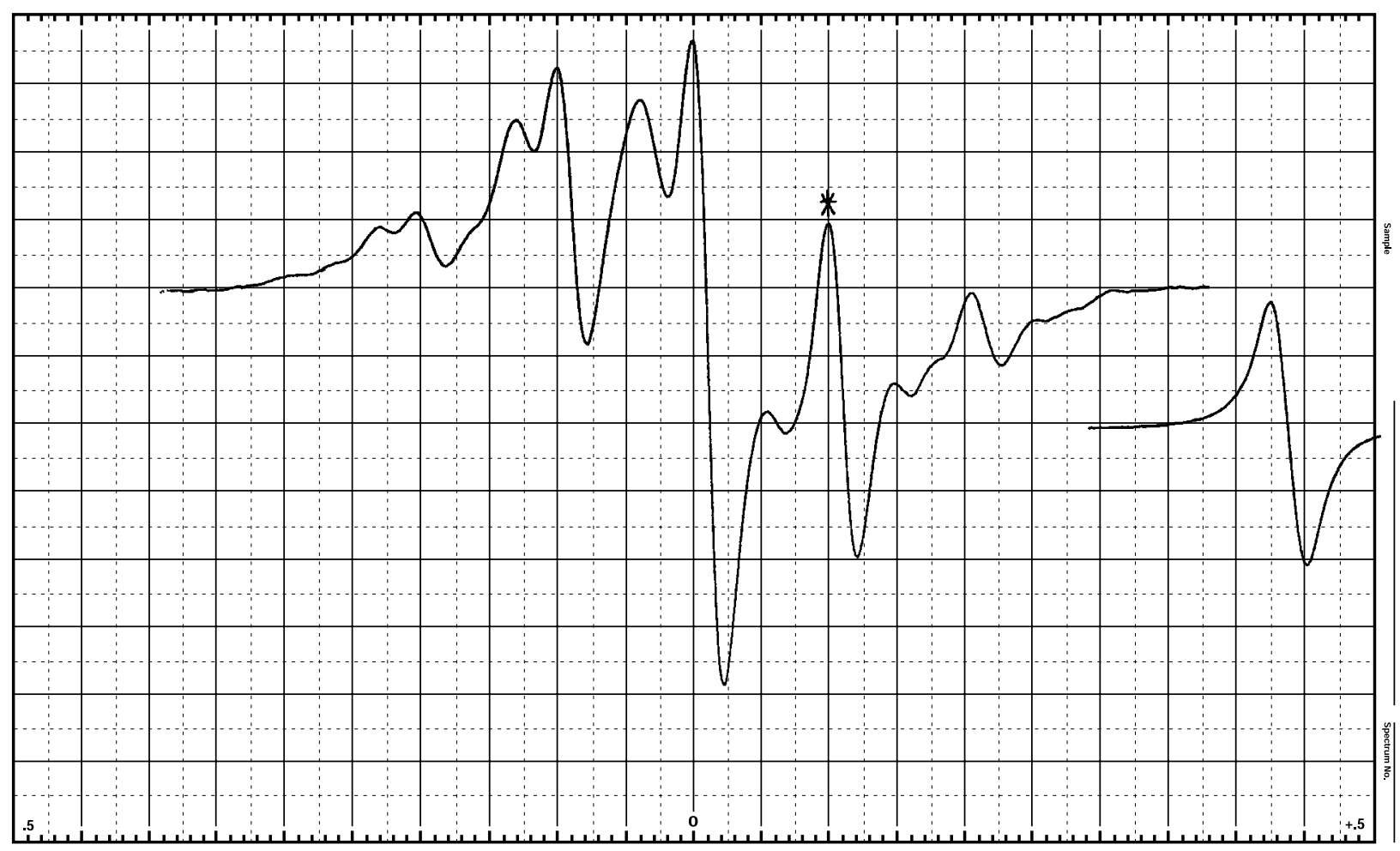

Figure 1 ESR spectrum of a gamma-irradiated PMAN sample at vacuum.

9100 with a TA-9900 data processing system, under nitrogen atmosphere and a heating rate of $10^{\circ} \mathrm{C} / \mathrm{min}$.

\section{RESULTS AND DISCUSSIONS}

\section{Kinetics}

In the previous studies, ${ }^{9,10}$ it was shown that the chain scission would happen in PMAN when it is exposed to high-energy radiation. This result confirmed by observing the complete solubility of the gamma-irradiated PMAN in acetone at room temperature. The 13-line ESR spectrum of irradiated PMAN sample presented in Figure 1. The intensities and places of ESR signals of the irradiated samples didn't change when they were kept for a long time under vacuum and at room temperature. This means that the radical transfers completed during the gamma irradiation with relatively low dose rate, and the radicals in the structure reached a steady state at room temperature. When the sample irradiated under vacuum was exposed to oxygen, the intensity of ESR peaks started to decrease; and after a long time of wait- ing in air, the shape of the spectrum turned to the asymmetric peroxy radical line (Fig. 2). For that reason, the decay of the radicals was only resulted from oxygen molecules, which permeated into the structure, after exposing them to air at room temperature. The self-decay reaction of radicals was only observed under vacuum when the tempera-

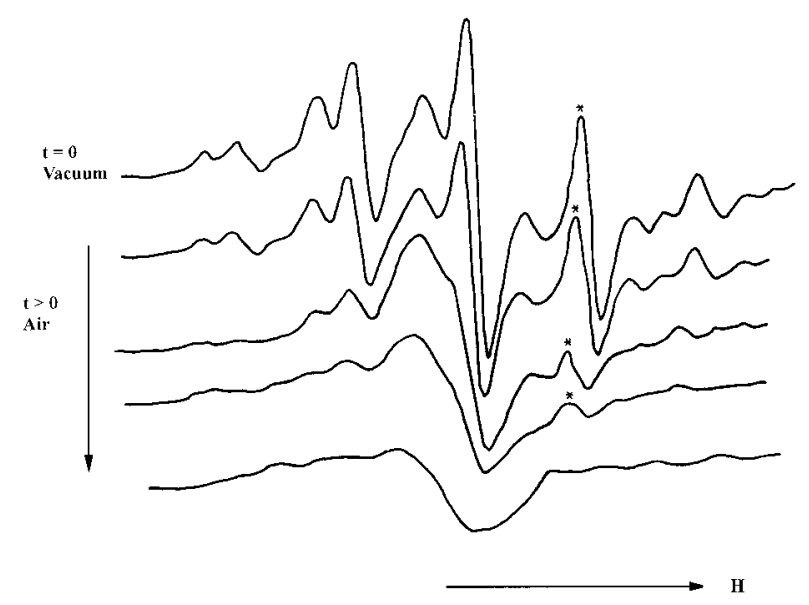

Figure 2 ESR spectrum of the asymmetric peroxy radical. 


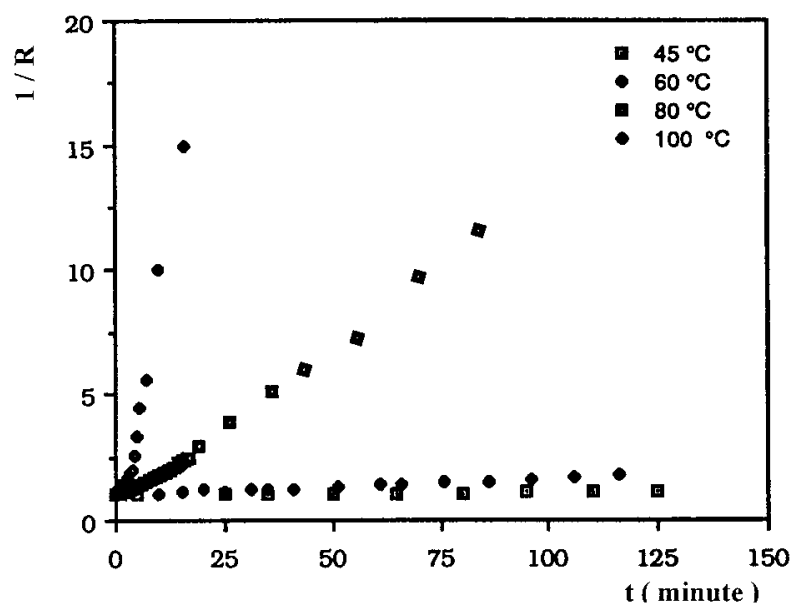

Figure 3 The change of normalized radical concentration $(1 / R)$ with time of the $36 \mathrm{kGy}$-gamma-irradiated sample at different temperatures, while keeping the samples under vacuum conditions.

ture raised due to the increase of the movement of the polymer chains. This termination of radicals under vacuum can take place within to different ways mentioned above.

As the radical concentration $(R)$, the value of the peak height with asterisk in Figure 1 taken as a function of time was used after normalization by dividing the DPPH peak values taken just after the ESR spectrum of the PMAN sample. The reason of the selection of the peak with the asterisk was that it did not overlap with peroxy radical, and there was no change on the width of this peak in accordance with the previous studies. ${ }^{1,7,8}$

To understand the radical termination reactions kinetics and degrees, the relation between radical concentration $(R)$ and time was investigated, and a linear relation was obtained between $1 / R$ and the time for termination reactions both in air and under vacuum at different temperatures in Figures 3-5. This result showed that the kinetics of the termination reactions obeyed the second degree reaction mechanism, and the following equation could be written for both air and vacuum termination reactions:

$$
\text { Rate }=-(d R / d t)=\mathrm{k}[R]^{2}
$$

When the termination in vacuum was considered, if $\mathrm{k}_{\mathrm{vac}}$ is the rate constant of the self-decay reaction under vacuum, the radical concentrations at time $t=0$ and $t=t$ are shown as $R_{0}$ and $R_{t}$, respectively. Equation (1) can be rewritten as follows after having an integral:

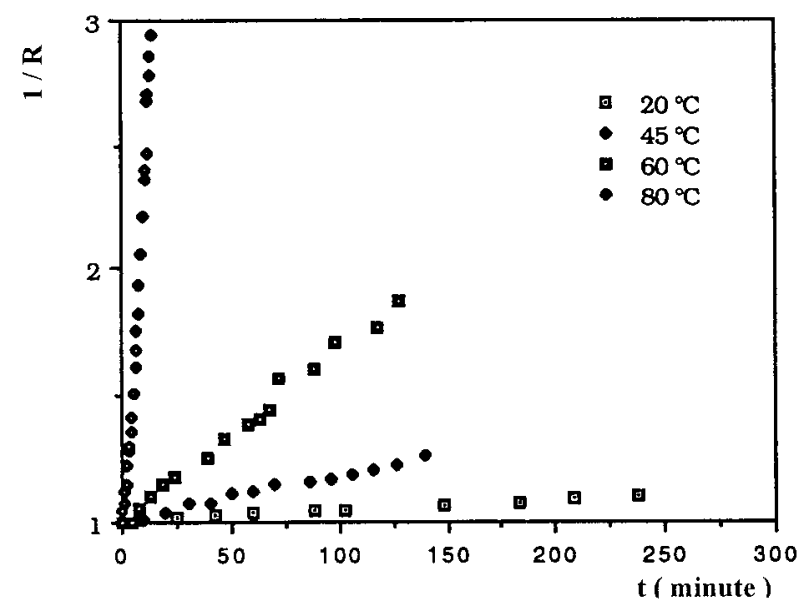

Figure 4 The change of normalized radical concentration $(1 / R)$ with time of the $36 \mathrm{kGy}$-gamma-irradiated sample from 20 to $80^{\circ} \mathrm{C}$ after exposing the samples to air.

$$
\mathrm{l} / R_{t}-\mathrm{l} / R_{0}=\mathrm{k}_{\mathrm{vac}} t
$$

The $\mathrm{k}_{\mathrm{vac}}$ values of the vacuum termination reactions at a temperature range from 45 to $100^{\circ} \mathrm{C}$ for the cylindrical PMAN samples irradiated at a $36-k$ Gy radiation dose were calculated from the slopes of the lines in the $(1 / R)$ - time, shown in Figure 3 and presented in Table I.

Total radical consumption in air at a raised temperature is the total of the oxygen effect termination reaction and the self-decay termination reaction, like in vacuum. For that reason, the termination reaction rate that resulted from only

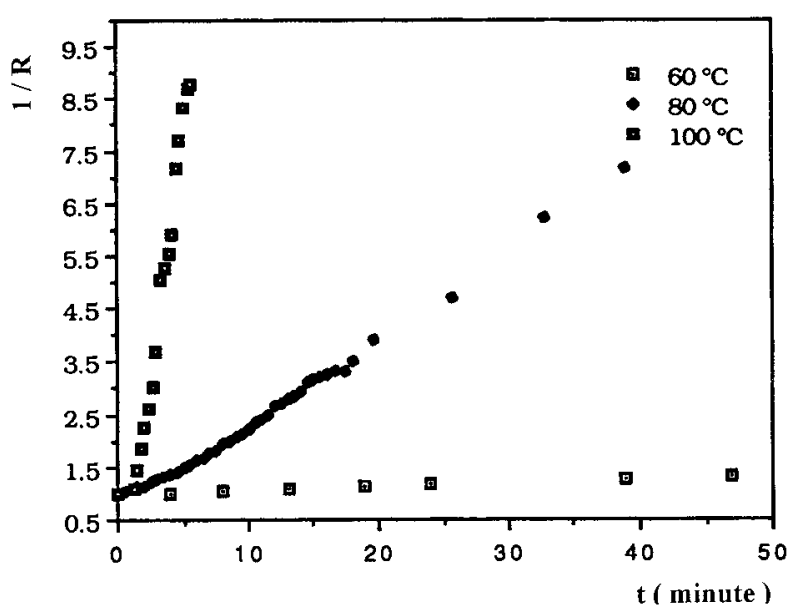

Figure 5 The change of normalized radical concentration $(1 / R)$ with time of the $36 \mathrm{kGy}$-gamma-irradiated cylindrical samples from 60 to $100^{\circ} \mathrm{C}$ after exposing the sample to air. 
Table I The Rate Constants (k) of the Radical Termination Reactions Under Vacuum, in Air, and Due to Oxygen at Different Temperatures

\begin{tabular}{cccc}
\hline $\begin{array}{c}\text { Temperature } \\
\left({ }^{\circ} \mathrm{C}\right)^{\mathrm{a}}\end{array}$ & $\mathrm{k}_{\mathrm{vac}}$ & $\mathrm{k}_{\text {air(tot })}$ & $\mathrm{k}_{\mathrm{O}^{2}}$ \\
\hline 25 & None & $0.49 \times 10^{-5}$ & $0.49 \times 10^{-5}$ \\
45 & $3.24 \times 10^{-5}$ & $4.54 \times 10^{-5}$ & $1.3 \times 10^{-5}$ \\
60 & $17.5 \times 10^{-5}$ & $24.6 \times 10^{-5}$ & $7.1 \times 10^{-5}$ \\
80 & $108 \times 10^{-5}$ & $164 \times 10^{-5}$ & $56 \times 10^{-5}$ \\
100 & $324 \times 10^{-5}$ & $708 \times 10^{-5}$ & $384 \times 10^{-5}$ \\
\hline
\end{tabular}

${ }^{\text {a }}$ Samples were irradiated at $36 \mathrm{kGy}$ under vacuum.

oxygen during high-temperature diffusion could be calculated from the difference of the reaction rates between the air termination rate and the vacuum termination rate, as follows:

$$
\begin{gathered}
\sim \mathrm{R}^{\bullet}+\mathrm{O}_{2} \stackrel{\mathrm{K}_{\mathrm{O}^{2}}}{\longrightarrow} \sim \mathrm{ROO}^{\bullet} \\
\sim \mathrm{R}^{\bullet}+\sim \mathrm{R}^{\bullet} \mathrm{R}^{\bullet} \stackrel{\mathrm{k}_{\mathrm{vac}}}{\longrightarrow} \text { Polymer } \\
(-d R / d t)_{O^{2}}=(-d R / d t)_{\mathrm{air}}-(-d R / d t)_{\mathrm{vac}} \\
(-d R / d t)_{O^{2}}=\mathrm{k}_{\mathrm{O}^{2}}[R]\left[O_{2}\right]
\end{gathered}
$$

The concentration of the reacted oxygen could be assumed as equal to the radical concentration that the oxygen reacted with it because the oxygen concentration on the surface of the polymer sample was always stable and there were an excess of oxygen from air during all of the diffusion process. When the oxygen concentration was replaced with the radical concentration, eq. (4) was rewritten as below.

$$
(-d R / d t)_{O^{2}}=\mathrm{k}_{\mathrm{O}^{2}}[R]^{2}
$$

If the rate constant of the total termination reaction in air is shown as $\mathrm{k}_{\text {tot }}$, and eqs. (1) and (5) were combined with eq. (3), the following equations could be formed.

$$
\begin{aligned}
(-d R / d t)_{\text {air }}=\mathrm{k}_{\mathrm{O}^{2}}[R]^{2}+\mathrm{k}_{\mathrm{vac}}[R]^{2} \\
\quad=\left(\mathrm{k}_{\mathrm{O}^{2}}+\mathrm{k}_{\mathrm{vac}}\right)[R]^{2}=\mathrm{k}_{\mathrm{tot}}[R]^{2}
\end{aligned}
$$

The experimental results of the air termination, which gave a linear relation between $(1 / R)$ and time in Figures 4 and 5 verified the above result that air termination was also a second-order kinetic reaction. After the integral of eq. (6), it became

$$
\mathrm{l} / R_{t}-\mathrm{l} / R_{0}=\mathrm{k}_{\mathrm{tot}} t
$$

In order to calculate $\mathrm{k}_{\text {tot }}$ of the radical termination reaction, irradiated cylindrical PMAN with $36 \mathrm{kGy}$ of gamma rays were exposed to air at a temperature range from room temperature to $100^{\circ} \mathrm{C} .1 / R$ values obtained from the normalizedheight values of the peak with an asterisk in Figure 1 were plotted against time in Figures 4 and $5 . \mathrm{k}_{\text {tot }}$ 's were calculated from the slope values of these curves in those figures and tabulated in Table I.

The rate constants of the termination reaction, which could result only from oxygen diffusion for raised temperature were calculated from the difference between $\mathrm{k}_{\text {tot }}$ and $\mathrm{k}_{\mathrm{vac}}$ in accordance with eq. (6). These $\mathrm{k}_{\mathrm{O}^{2}}$ values were presented in Table I.

It can be seen from Table I that $\mathrm{k}$ values increased with increasing temperature. The reason for this increase was thought to be the increase of chain mobility, which provides the macro radicals with the self-decay reaction and increases the diffusion rate of oxygen through the increase of the free volume in the structure and thermal movement of the polymer chains.

The activation energies of the three-termination reactions were calculated from the Arhenius equation, as follows:

$$
\mathrm{k}=\mathrm{A} e^{-E \alpha / \mathrm{R} T}
$$

where $\mathrm{A}$ is constant; $E_{a}$, the activation energy; R, the gas constant; and $T$, temperature. $\ln \mathrm{k}$ values for each termination reaction were plotted against 1/T values to calculate $E_{a}$ values for them. 


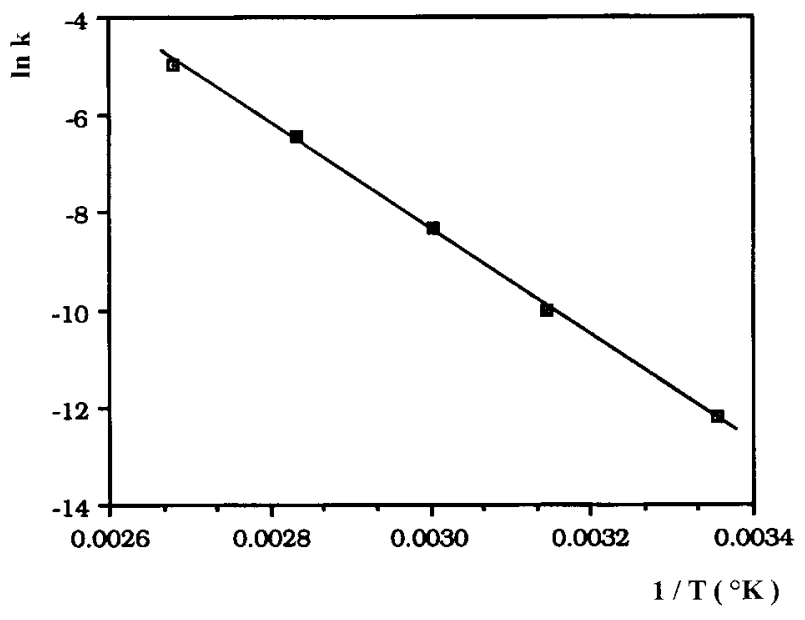

Figure 6 The change of $\ln k$ with $1 / T$ of the radical termination reaction of gamma-irradiated cylindrical samples after exposing the samples to air.

They were shown in Figure 6 for the air termination reaction only and in Figure 7 for three three reactions together. The activation energies were found $88.39,83.22$, and $81.39 \mathrm{~kJ} / \mathrm{mol}$ for air, the oxygen effect, and vacuum termination reactions, respectively.

\section{Diffusion}

The cylindrical samples were irradiated with different dose values at room temperature under vacuum to calculate the zero-dose diffusion coefficient $(D)$ and evaluate the relation between $D$ and dose. After having exposed the irradiated sample to air at room temperature, the changes of the peak with an asterisk in Figure 1 were observed for a half-an-hour time interval at the beginning, and then durations extended to a longer time period, like 1 day, to obtain the change between the ESR spectrums. The ESR peak values of PMAN were normalized by dividing the peak values of the DPPH's ESR spectrum, which has been taken just after PMAN samples.

When a very long time is necessary for saturation with oxygen of the relatively big cylindrical samples, eq. (9), derived from Fick's second diffusion law under the following two conditions, can be written to calculate the diffusion coefficient. ${ }^{12,13}$ First,

$$
\begin{array}{ccc}
t=0 & a<r<0 & c=0 \\
t>0 & r=a & c=c_{0}
\end{array}
$$

where $a$ is the radius of cylindrical sample, and $r$ is any point on $a$ on which diffusion takes place.

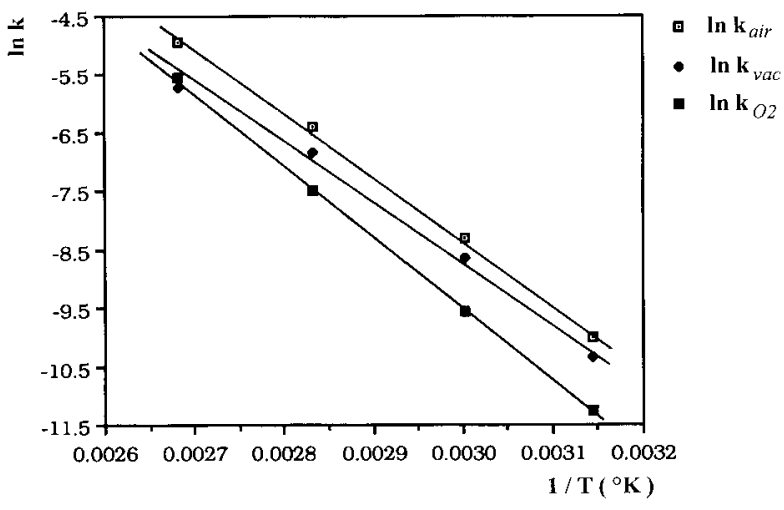

Figure 7 The change of $\ln k$ with $1 / T$ of the radical termination reaction under vacuum, in air, and due to the oxygen effect only, respectively.

That means that before diffusion starts into structure $(t=0)$, the concentration of the gases in the structure should be zero $(c=0)$, and during the diffusion processes $(t>0)$, the concentration of the gases that contact on the surface of the sample should not change $\left(c=c_{0}\right)$.

Second, the diffusion which takes place from the sides of cylinder through radius, can be only considered for calculation, and the diffusion from the ends of cylinder can be small enough to be neglected, which is possible when the height of cylinder is bigger enough than radius.

$$
M_{t} / M_{\infty}=1-\sum\left\lfloor 4 / \alpha_{n}^{2} a^{2}\right\rfloor \exp \left\lfloor-D \alpha_{n}^{2} t\right\rfloor
$$

where $M_{t}$ and $M_{\infty}$ are the masses of the gases, which penetrate into polymer at time $(t)$ and infinity, respectively. The first four values of $\alpha_{n}$ were given as $\propto_{1}=2.404 / a, \alpha_{2}=5.520 / a, \propto_{3}$

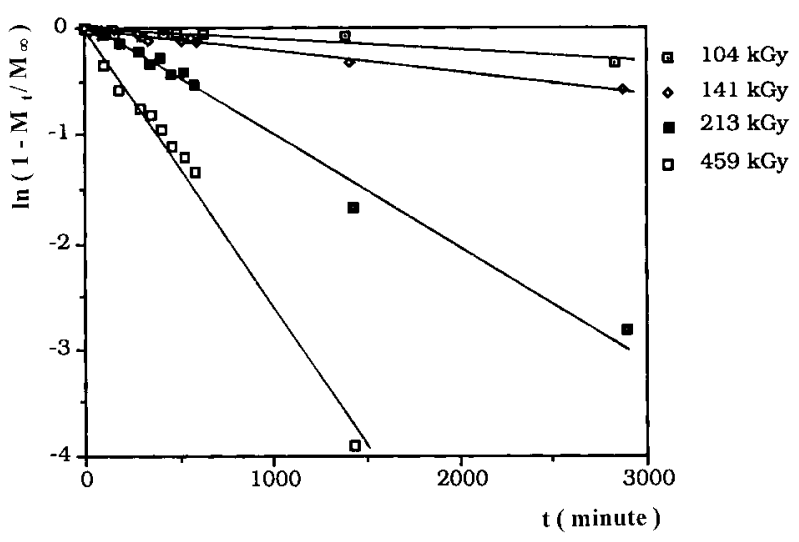

Figure 8 The change of $\ln \left(1-M_{1} / M_{\infty}\right)$ with time of samples irradiated at high dose values. 


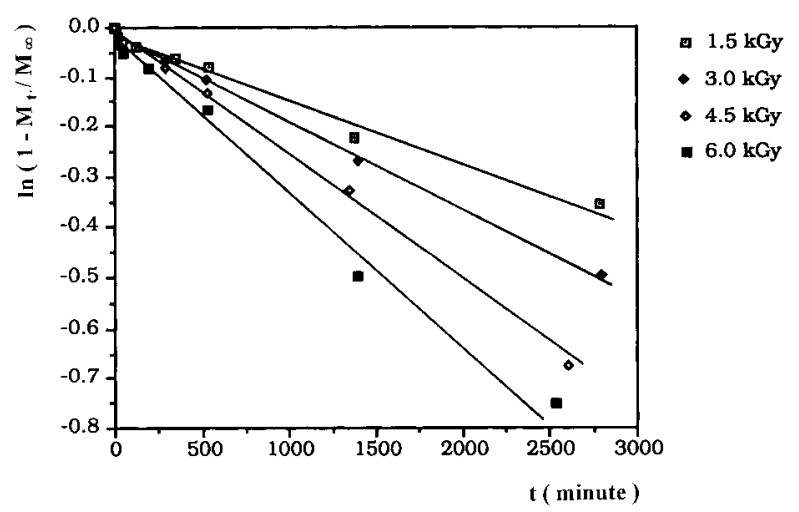

Figure 9 The changes of $\ln \left(1-M_{t} / M_{\infty}\right)$ with time of samples irradiated at low dose values.

$=8.654 / a$, and $\propto_{4}=11.790 / a .{ }^{13}$ Since the diffusion took place in a very long time due to the fact that the sample sizes were relatively big cylinders in this study, $\propto_{1}>1$ values could be omitted with negligible error, and eq. (9) was simplified as follows ${ }^{13}$ to calculate the diffusion coefficient:

$$
M_{t} / M_{\infty}=1-\left\lfloor 4 / \alpha_{1}^{2} a^{2}\right\rfloor \exp \left\lfloor-D \alpha_{n}^{2} t\right\rfloor
$$

After exposing the irradiated sample to air, the change of the ESR signal was followed by time, and $R_{t}$ and $R_{0}$ values were calculated from the heights of the peak with an asterisk in Figure 1 at time $t=t$, and for the same sample, time $t=0$. $\ln \left(1-M_{t} / M_{\infty}\right)$ values were calculated from $\left(R_{t} /\right.$ $R_{0}$ ) values using eq. (11) in accordance with the previous studies. ${ }^{1,7,8}$ This equation could be reobtained for the cylindrical system when the side surface of the samples is bigger enough than the top and bottom surface of the cylindrical samples in order to able to accept the diffusion from only radial $(r)$ into polymer with negligible errors.

$$
1-M_{t} / M_{\infty}=R_{t} / R_{0}
$$

$\ln \left(1-M_{t} / M_{\infty}\right)$ values of irradiated PMAN samples with high-dose values and low-dose values were plotted against time $(t)$ separately in Figures 8 and 9 , respectively. The diffusion coefficient $(D)$ of cylindrical PMAN samples for each dose values was calculated from the slope of the corresponding curve in Figures 8 and 9 by using eq. (10). The $D$ values and the corresponding dose values were tabulated in Table II, and $D$ values were plotted against the dose in Figure 10. As can be seen from Figure 10, $D$ values increased with
Table II Diffusion Coefficients of Cylindrical PMAN Samples and Corresponding Dose

\begin{tabular}{|c|c|c|c|}
\hline $\begin{array}{c}\text { Dose (kGy) } \\
\text { (Low) }\end{array}$ & $\begin{array}{c}D \\
\left(\mathrm{~cm}^{2} / \mathrm{s}\right) 10^{-9}\end{array}$ & $\begin{array}{c}\text { Dose (kGy) } \\
\text { (High) }\end{array}$ & $\begin{array}{c}D \\
\left(\mathrm{~cm}^{2} / \mathrm{s}\right) \\
10^{-9}\end{array}$ \\
\hline 1.5 & 3.6 & 104 & 10.6 \\
\hline 3.0 & 4.3 & 141 & 12.1 \\
\hline 4.5 & 4.8 & 213 & 38.4 \\
\hline 6.0 & 5.0 & 459 & 195 \\
\hline
\end{tabular}
Values (Room Temperature)

the increasing irradiation dose values, which means that the radiation dose accelerated the diffusion mechanism, contrary to the previous observation ${ }^{1,7}$ but in accordance with expectations.

Since the relation between $D$ and the dose was not linear in Figure 10, $\ln D$ was plotted against the dose in order to find the dose-independent diffusion coefficient $\left(D_{0}\right)$ (Fig. 11). The low-dose region of this curve was extrapolated to the zerodose value, and $D_{0}$ for the cylindrical PMAN sample was calculated as $D_{0}=3.1 \times 10^{-9} \mathrm{~cm}^{2} / \mathrm{s}$.

Although the dependence of the diffusion to dose for relatively big cylindrical samples was completely reverse, the ones of the thin film and tablet samples, as mentioned above, there was very good agreement between the dose-independent diffusion coefficient value of cylindrical samples and the previous ones, for which the $D_{0}$ values of the thin film and tablet were found 2.8 $\times 10^{-9}$ and $2.7 \times 10^{-9} \mathrm{~cm}^{2} / \mathrm{s}$ respectively. ${ }^{1}$ This result supported the explanation of the dose dependency of the oxygen diffusion into PMAN that

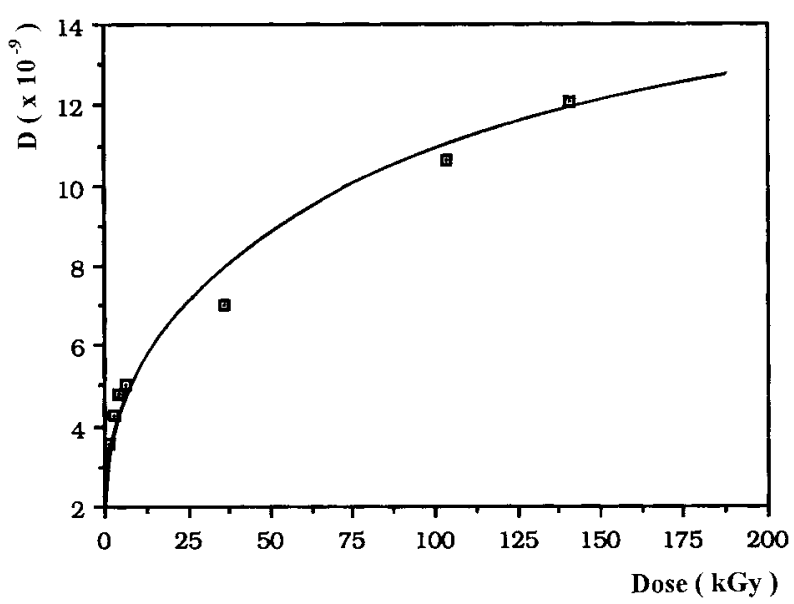

Figure 10 The change of $D$ with the radiation dose of the cylindrical samples irradiated at different dose values. 


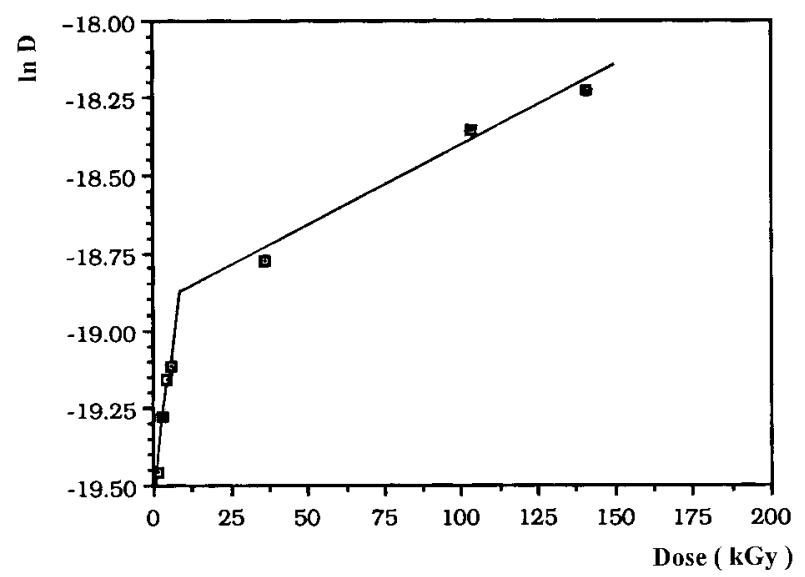

Figure 11 The change of $\ln D$ with the radiation dose of the cylindrical samples irradiated at different dose values.

is closely related to the sample shape from the standpoint of its surface/mass ratio.

According to the our previous explanation, when the polymer sample is irradiated with highenergy radiation, there will be the following two different effects from radiation to oxygen diffusion into polymer: while one effect decreases the diffusion, the other effect will increase the diffusion rate simultaneously. The net effect of radiation to diffusion will be determined from the difference between these effects.

The first effect from increasing radiation dose results in a decrease of the diffusion, like the diffusion into thin film, in which the surface/mass ratio was very big and the diffusion was very fast. The second effect was an increase in the diffusion rate with an increasing radiation dose like relatively big cylindrical samples, in which the surface/mass ratio was very much smaller than for thin film samples, and the diffusion took place very slowly.

In the case of the diffusion into surface dominating samples, which means that the surface/ mass ratio is very big and the diffusion completion happens in a short time, the peroxy radicals on the surface of the sample occupy some of the free volume to be used for the next oxygen molecules to permeate into structure. When the radiation dose increases, the number of the surface peroxy radicals' increase and the amount of the free volume on the surface decreases. As a result of this decrease of free volume, the diffusion of oxygen into the mass of the polymer sample will be difficult and slow, resulting in a decrease of the $D$ value.
In the case of the diffusion into mass dominating polymer samples, which means that the surface/mass ratio is very small and the diffusion takes place for a long time, the surface peroxy radical effect prevails by the following diffusionacceleration effect of the radiation dose.

1. Subject to eq. (1), any increase of the radical concentration in the whole structure will increase the velocity of the peroxy formation reaction and diffusion.

2. If chain scission takes place in the polymer sample with high-energy radiation, as being for PMAN, when the radiation dose increases, the number of the chain ends, which have a very important effect on the transportation of gases in the structure, will increase.

3. The increase of the free volume results from removing some gases from the structure during radiation process.

As a result, it can be said that the radiation effect on diffusion for polymeric samples is closely dependent on the surface/mass ratio of the sample. The following exponential equation can be rewritten to show dose dependence of the diffusion of the materials, which react with radicals like oxygen.

$$
D=D_{0} e^{j \propto t}
$$

where $\propto$ is a constant to make the ratio equal, and $j=-1$ or +1 , dependent on the dose effect that

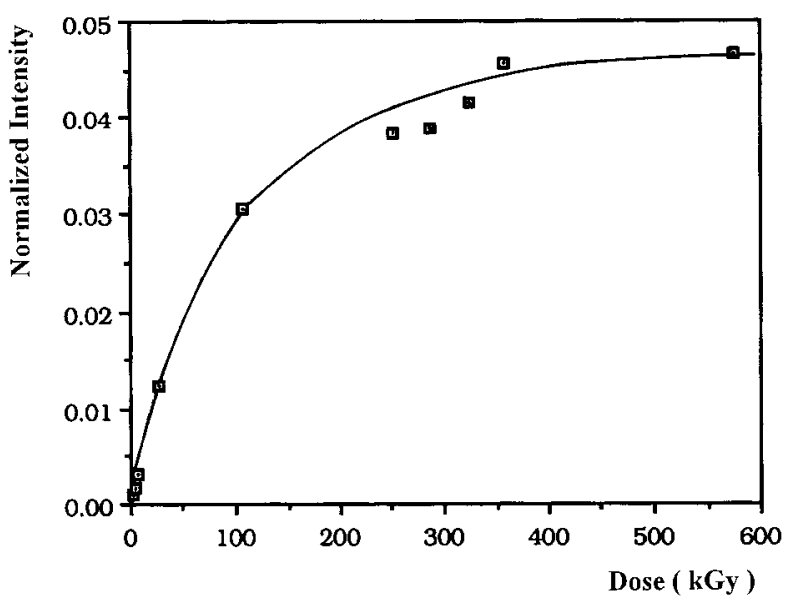

Figure 12 The total-intensity change of radicals in the irradiated samples with the radiation dose for saturation dose observation. 


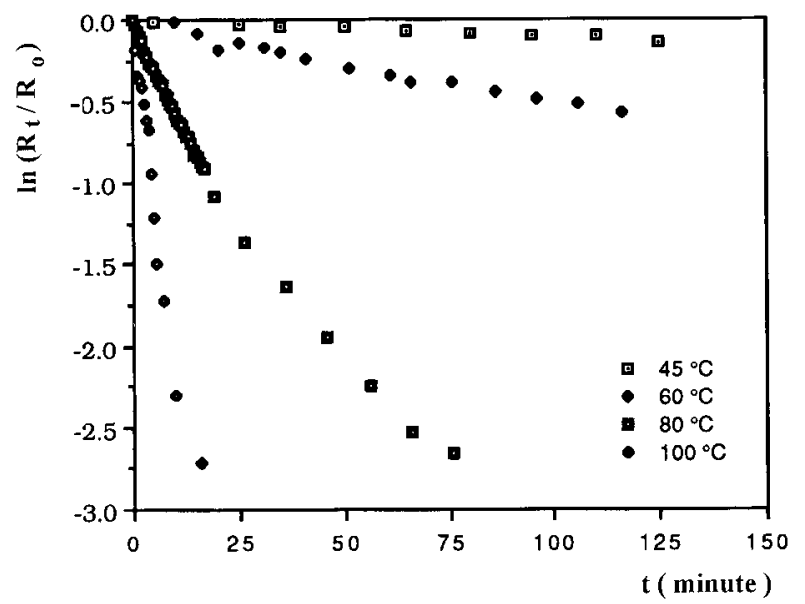

Figure 13 The change of $\ln \left(R / R_{0}\right)$ under vacuum with the time of the irradiated samples at a range of temperature from 45 to $100^{\circ} \mathrm{C}$.

can be changed with the surface/mass ratio of the samples irradiated. $j$ will likely be $j=+1$ for samples that have the very small values of surface/mass, like samples used in this study; or $j$ is $j=-1$ for samples that have very big values of surface/mass, like very thin film samples used for the previous study. ${ }^{1}$ The deviation for this exponential relation will likely take place when the radiation dose values are high enough to be near to the saturation-dose values of the sample, which the radical concentration doesn't increase proportionally with the dose increase as in Figure 12. This can be observed as a second slope in the ln $D$-dose relation, as in Figure 11.

\section{The Diffusion Activation Energy}

In order to calculate the diffusion activation $\left(E_{a}\right)$ energy of PMAN, the cylindrical samples were

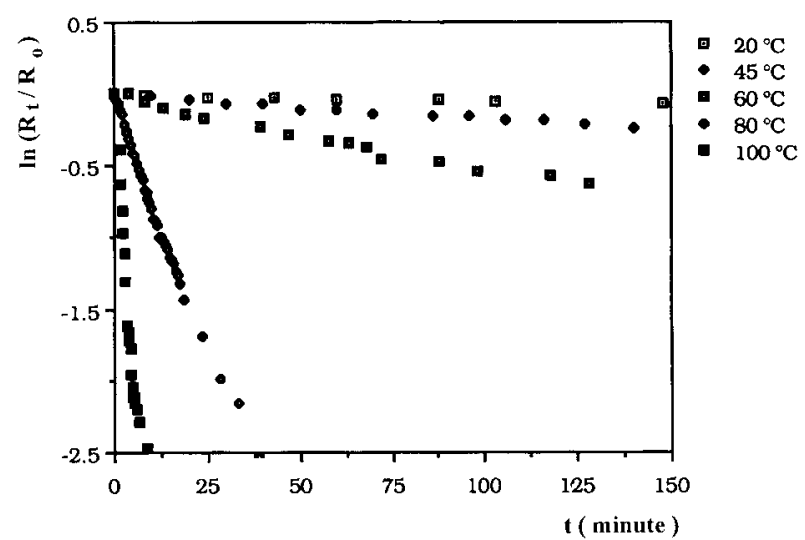

Figure 14 The change of $\ln \left(R / R_{0}\right)$ in air with the time of the irradiated samples at a range of temperature from 20 to $100^{\circ} \mathrm{C}$.
Table III Diffusion Coefficients of Cylindrical PMAN Samples Irradiated at 36 kGy Under Vacuum and Corresponding Temperature Values

\begin{tabular}{rl}
\hline$T\left({ }^{\circ} \mathrm{C}\right)$ & $D\left(\mathrm{~cm}^{2} / \mathrm{s}\right)$ \\
\hline 25 & $9.2 \times 10^{-9}$ \\
45 & $7.2 \times 10^{-8}$ \\
60 & $9.0 \times 10^{-8}$ \\
80 & $2.0 \times 10^{-6}$ \\
100 & $2.7 \times 10^{-5}$ \\
\hline
\end{tabular}

irradiated with a 36 -kGy dose value at room temperature under vacuum, for which they were used for the above-mentioned kinetic study. After having exposed the irradiated samples to air, the change of the ESR peak with the asterisk in Figure 1 was followed at the room temperature (25), $45,60,80$, and $100^{\circ} \mathrm{C}$ under isothermal conditions. Since the self-decay of the radicals was observed for vacuum at $45^{\circ} \mathrm{C}$ and above, the ESR peak change under vacuum was observed for these temperature values in order to find out the change that resulted from only oxygen diffusion. $\ln \left(R / R_{0}\right)$ were plotted against time for both the samples exposed air and those samples kept under vacuum at the same temperature for Figs. 13 and 14. The difference of the slopes between air exposed and vacuum-waited samples at the same temperature was used as the slope of net oxygen diffusion event in the equation 14 and $D$ values for each temperature was calculated and presented in the Table III. The following Arhenius equation was used in order to find the activation energy of diffusion; and in accordance with this equation, $\ln D$ values were plotted against $1 / T$ (Fig. 15).

$$
D=\mathrm{A} e^{-E \alpha / R T}
$$

As it can be seen from Figure 15, the slope of the curve between room temperature and $60^{\circ} \mathrm{C}$ was different from the slope values above $60^{\circ} \mathrm{C}$, which means that two different values of the activation energy of the diffusion into PMAN. The first $E_{a 1}$ was calculated as $27.9 \mathrm{~kJ} / \mathrm{mol}$ for below $60^{\circ} \mathrm{C}$, and the second $E_{a 2}$ was calculated as $74.2 \mathrm{~kJ} / \mathrm{mol}$ for the above $60^{\circ} \mathrm{C}$. The reason of these two-different activation energies was thought from the difference of the diffusion mechanism of oxygen into the polymer below and above $60^{\circ} \mathrm{C}$. 


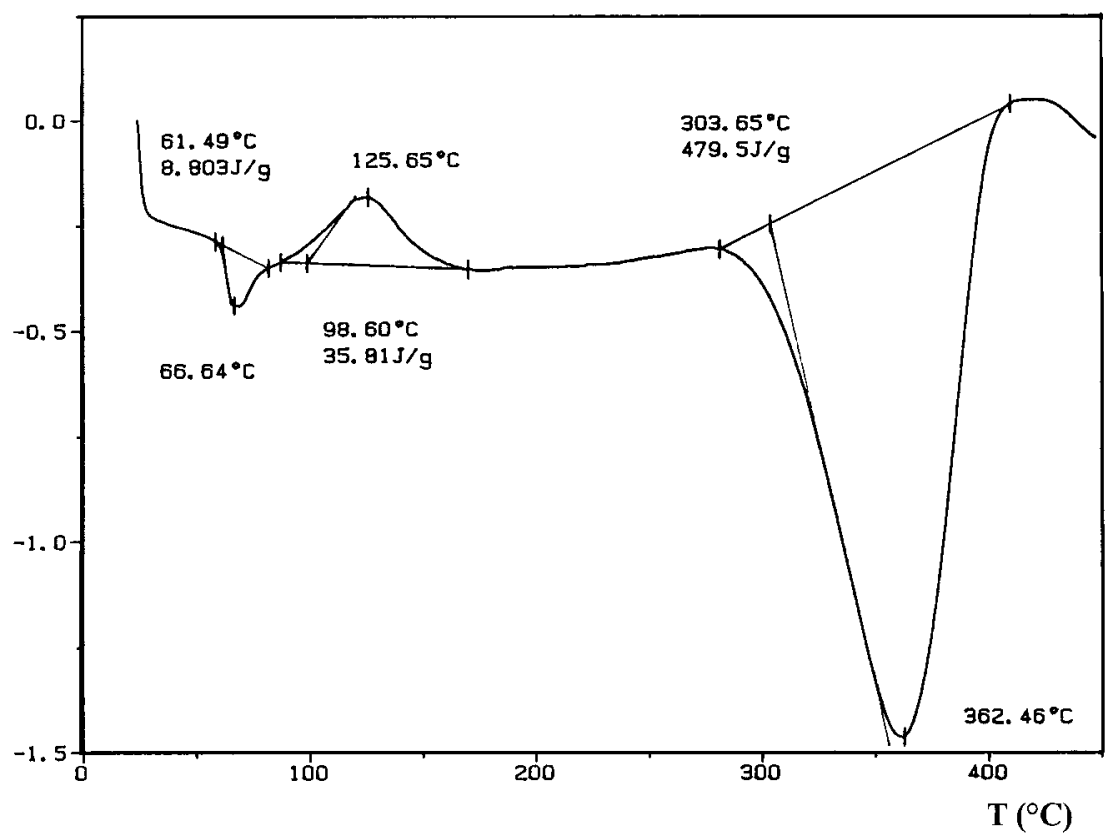

Figure 16 The DSC thermogram of PMAN.

To understand if any thermal change occurs around this temperature, DSC thermograms of the unradiated PMAN sample were taken (Fig. 16). As it can be seen from Figure 16, there was an endothermic event about $65^{\circ} \mathrm{C}$, which after preheating to $100^{\circ} \mathrm{C}$, turned to a baseline drop (Fig. 17). The first endothermic peak was evaluated from the relaxation energy effect in Figure $17(\mathrm{a})$, and the baseline drop at about $65^{\circ} \mathrm{C}$ in Figure 17(b) was most likely the $T_{g}$ value of

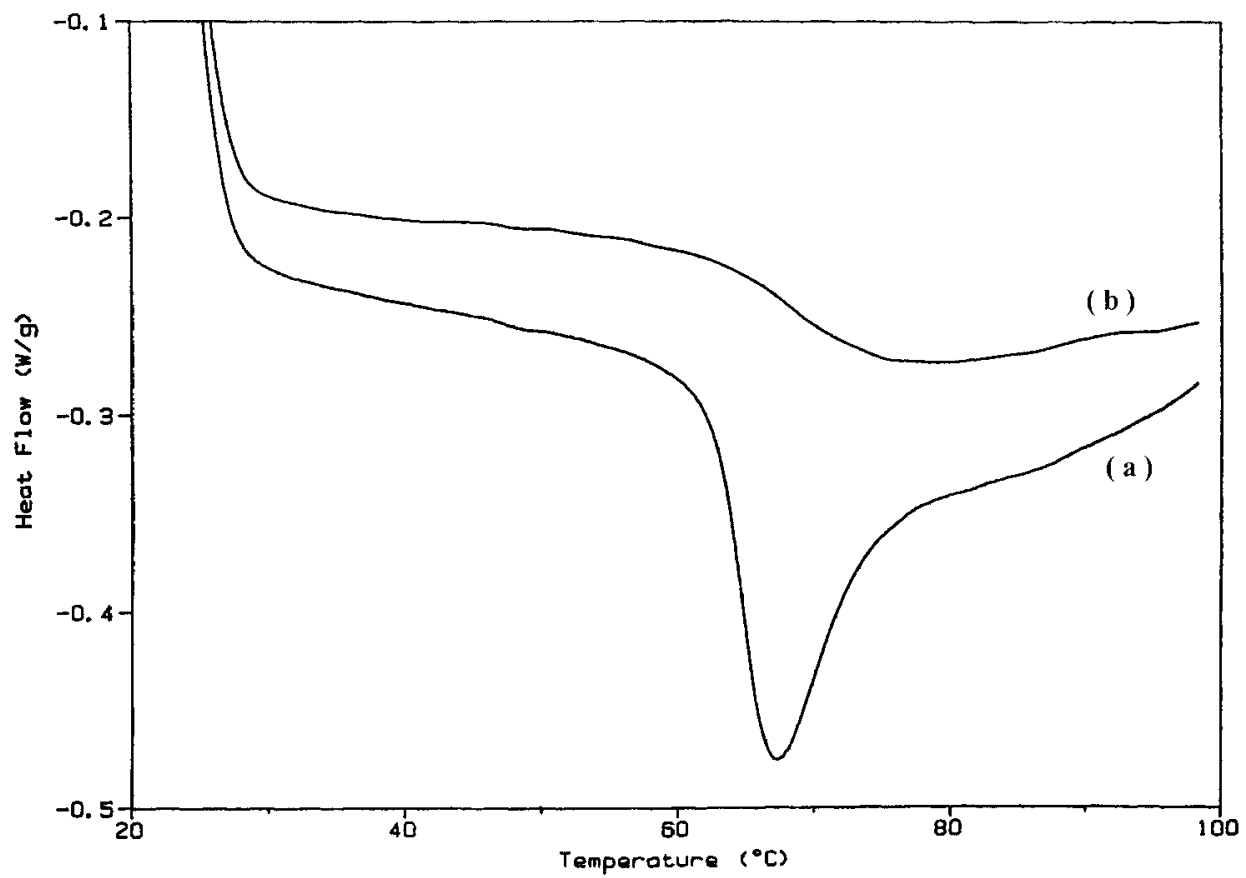

Figure 17 The DSC thermogram of PMAN: (a) the original sample; (b) the preheated sample to $100^{\circ} \mathrm{C}$. 


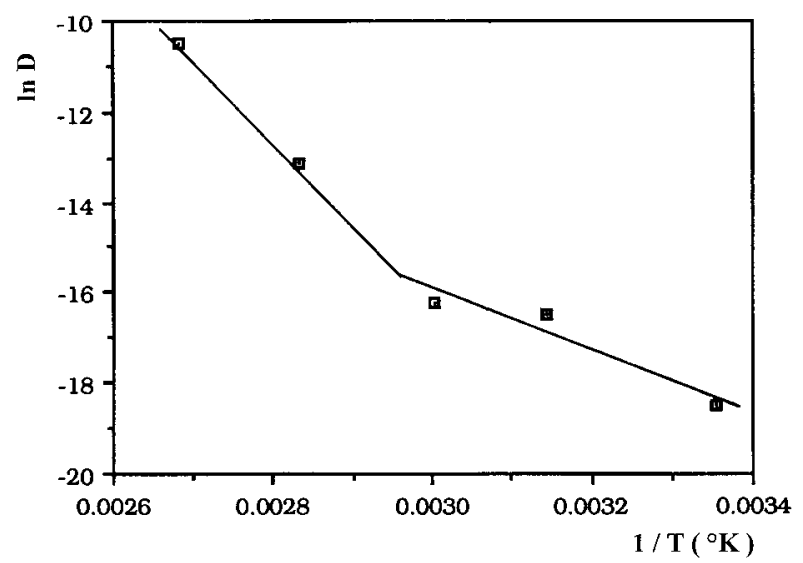

Figure 15 The change of $\ln D$ with $1 / T$ of the PMAN.

the PMAN. ${ }^{2}$ The temperature value $\left(60^{\circ} \mathrm{C}\right)$ of $E_{a}$ change was very close to this temperature $\left(65^{\circ} \mathrm{C}\right)$. On the other hand, $65^{\circ} \mathrm{C}$ was quite different from the literature $T_{g}$ values of PMAN given above $100^{\circ} \mathrm{C} .{ }^{14,15}$ To understand the reason of the $T_{g}$ 's value difference and to find out the exact $T_{g}$ value of the PMAN sample used in this study, a deep $T_{g}$ study has been done and will be ready for publication soon. ${ }^{2,16}$

The activation energy difference could be explained as more of an energy requirement $\left(E_{a 2}\right.$ $=74.2 \mathrm{~kJ} / \mathrm{mol}$ ) for the diffusion above the $T_{g}$ temperature because of the increase of the molecular movement of polymer above $T_{g}$, which is a very important effect on the diffusion. Activating more movement of the polymer chains through the temperature rise would require more energy than the ones that have lower mo- lecular movement from being below the $T_{g}$. This result could also be interpreted as the possibility of determination of $T_{g}$ value of the polymers through the diffusion coefficient change with temperature.

\section{REFERENCES}

1. Katircioglu, Y.; Kaptan, H. Y.; Guven, O. to appear.

2. Katircioglu, T. Y. Ph.D. Thesis, Hacettepe U. Ankara, 1992.

3. Michaels, A. S.; Bixler, H. J.; Fein, P. L. J Appl Phys 1964, 35, 3165.

4. Holden, P. S.; Orchard, G. A. J.; Ward, I. M. J Polym Sci, Polym Phys Ed 1985, 23, 709.

5. Migliaresi, M.; Nicodemo, L.; Nicolais, L.; Passerini, P. Polymer 1984, 25, 686.

6. Brandrup, J.; Immergut, E. H. Polymer Handbook; Wiley-Interscience: New York, 1989.

7. Kaptan, H. Y.; Pekcan, O.; Guven, O. J Appl Polym Sci 1992, 44, 1595.

8. Kaptan, H. Y.; Pekcan, O.; Arca, E.; Guven, O. J Appl Polym Sci 1989, 37, 2577.

9. Weiss, A. R.; Allen, A. D.; Schawarz, H. A. Proceedings of the International Conference on Peaceful Uses, New York, Vol. 14; 1956, p 179.

10. Schlick, S.; Kevan, L. J Appl Polym Sci 1982, 27, 319.

11. Helbert, J. N.; Poindexter, E. H. J Polym Sci, Polym Chem Ed 1979, 17, 49.

12. Crank, J. The Mathematics of Diffusion; Oxford University Press: London, UK, 1970.

13. Saw, G. Trans Faraday Soc 1967, 63, 2181.

14. Nakamura, S.; Otake, T.; Matsuzaki, K. J Appl Polym Sci 1972, 16, 1817.

15. Nelson, C. E. Mechanical Properties of Polymers; Reinhold: New York, 1962.

16. Katircioglu, Y.; Guven, O. to appear. 